\title{
CONDITIONAL MIXED-STATE MODEL FOR STRUCTURAL CHANGE ANALYSIS FROM VERY HIGH RESOLUTION OPTICAL IMAGES
}

\author{
Benjamin Belmudez $z^{1,2,3}$, Véronique Prinet ${ }^{1}$, Jian-Feng Yao ${ }^{2}$, Patrick Bouthemy ${ }^{2}$, Xavier Descombes ${ }^{3}$ \\ ${ }^{1}$ NLPR \& LIAMA, Institute of Automation, Chinese Academy of Sciences, Beijing 100190, P.R. China \\ ${ }^{2}$ Vista, IRISA/INRIA Rennes, Campus Beaulieu, 35042 Rennes Cedex, France \\ ${ }^{3}$ Ariana (joint research group INRIA/I3S), INRIA, Sophia-Antipolis, 06902 Cedex, France
}

\section{INTRODUCTION}

The advent of very high resolution optical satellite images has opened, since nearly one decade already, a whole range of new possibilities in application domains like digital cartography, urban planning or land survey. The variety and the amount of information available in these images make nevertheless classical techniques of classification and segmentation obsolete. High accuracy comes with high level of details, which are not necessarly relevant information and add 'geometric' noise of the image. The challenge is then to get rid of the 'noise' (car, road lines, vegetation,..), while exploiting valid and accurate information.

The present work concerns the analysis of dynamic scenes from earth observation images. More precisely, we are interested in building a map which, on one hand locates places of change, on the other hand, reconstructs a unique visual information of the non-change areas. To this end, we propose a new approach based on conditional mixed-state random field modeling (mixed-state CRF). The so-called "mixed-state" stems from the mixed nature of the unknown random variable: the discrete component is a binary indicator function of change v.s. non change, while the continuous component provides an estimation of the "background image" where no change happened. The maximum a posteriori (MAP) estimation of the CRF is, through the Hammersley-Clifford theorem, turned into an energy minimisation problem .

The design of the energy functional, the choice of appropriate features to describe the data, and the initialisation of the optimisation process are all introduced briefly in section 2. Results and conclusions are given in section 3 .

\section{CONDITIONAL MIXED STATE MODEL}

Markov Random Field models have been extensively used for various segmentation and labelling applications in vision. In MRF, the modeling task consists in defining a joint probability distribution $p(\mathbf{x}, \mathbf{y})$ over observed data $\mathbf{x}$ and unknown variables $\mathbf{y}$. Typically, in a generative framework and using the Bayes rule, the joint probability decomposes into an observation model (or likelihood) $p(\mathbf{x} \mid \mathbf{y})$ and a prior model $p(\mathbf{y})$. Facing the limitations of MRFs, Lafferty \& al. introduced in [1] the Conditional Random Field models. CRFs directly model the posterior probability of the unknown variable given the observation, i.e. $P(\mathbf{y} \mid \mathbf{x})$, without explicit modeling of the observation. It offers several advantages over MRFs: it drops the strong assumption of conditional independence of the data at each site ; it becomes possible to represent long range dependencies between observation and unknown variables.

CRF models until now were developed for either continuous or discrete random variables $[1,2]$. Conversely, inspired from [3], we are here interested in modelling a Gibbs energy functional $E_{G i b b s}(\mathbf{x}, \mathbf{y})$, where $\mathbf{y}=\left\{y_{i}\right\}_{i \in\{1, \ldots N\}}$ and $S \ni y_{i}$ a "mixed space" equipped with appropriate measure and defined by $S=\{w\} \cup \mathbb{R}^{+}$, with $w \in \Omega$ symbolic concepts that, in our case, takes negative integer values.

We incorporate mixed-state variables into CRF models. We propose a mixed-state energy functional expressed as the sum of a discrete term and a continuous term, associated respectively with the discrete component and the continous component of the random variable $\mathbf{y}$. We design this energy for applications to structural changes analysis. The observation $\mathbf{x}$ is described by radiometric and robust structural features based on local gradient histograms computed at local patches [4, 5]. We show that the optimal configuration $\tilde{\mathbf{y}}$ that minimises $E_{G i b b s}()$ can be retrieved by standard optimisation algorithms. We choose to use Iterated Conditional Mode, with careful initialisation, for its simplicity. This initial state results from a preliminary binary classification of the data generated by an Iterative Principal Component Analysis (IPCA, see [6]). Finally, $\tilde{\mathbf{y}}$ provides us with a map which, on one hand locates places of change (symbolic information), on the other hand, reconstructs a unique visual information of the non-change areas (quantitative measure). 


\section{RESULTS AND CONCLUSION}

Experimental tests were performed on several pairs of Quickbird panchromatic images ( $0.6 \mathrm{~m}$ resolution), covering the same geographical site (Beijing area). Pairs are assumed to be registered (via geo-coding in our case). While selecting test images, in purpose, we chose difficult cases which contain true structural changes (that we want to detect) and visual changes (cars, vegetation, projective effects) associated to "noise".

Figure 1 illustrates one of the results. Left images (a) show the two input data and the associated ground truth (in red). The computed result from mixed-state CRF is given in (b) extrem right : gray level values indicate the unchanged information contained in the two images, while in red color are pixels detected as change. From these results, it appears clearly that mixedstate CRF model performs better than traditional methods thanks to its capacity to take into account dependencies between observations at neighboor sites. We notice also that the stuctural descriptor plays an important role to withdraw false detections (in particular vegetation change). Remaining false positive detections are mainly due to projective effects of high buildings. Morphological filtering in a post-processing step could get rid of these errors.

The MSCRF model proposed in this paper could be improved in many ways. At first the unary term of the energy functional could be formulated in a more sophisticated fashion. In addition, one could further take advantage of the symbolic component of the unknow random variable and make use of it to classify different categories of change.
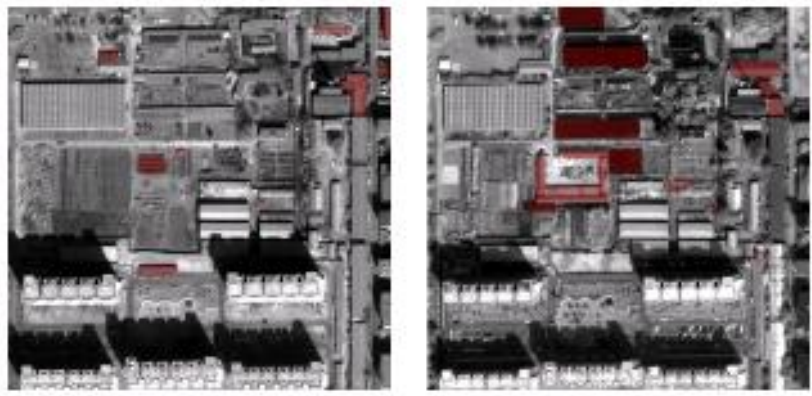

(a) Pair of Quickbird images (2001 and 2003) and manual labeling of structural changes (in red).
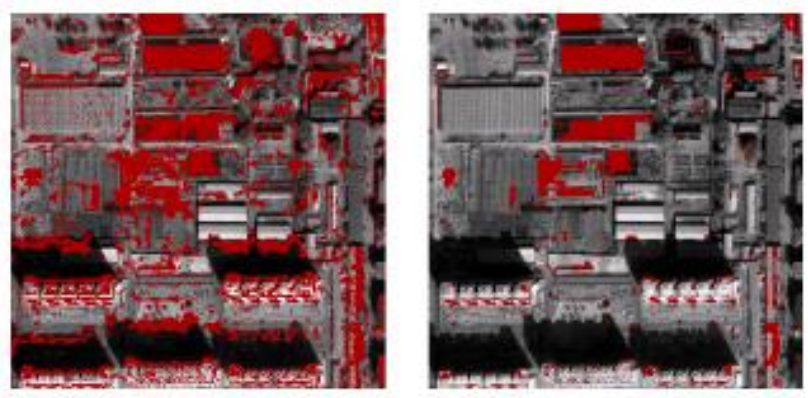

(b) Left: preprocessing from ICPA [6] ; Right: Change map (in red) and reconstructed visual image of non-changes.

Fig. 1. Change detection from Mixed-State Conditional Random Field Model

\section{REFERENCES}

[1] J. Lafferty, A. McCallum, and F. Pereira, "Conditional random fields: Probabilistic models for segmenting and labeling sequence data," in Proc. Int. Conf. on Machine Learning, Massachusetts, USA, 2001.

[2] S. Kumar and M. Herbert, "Discriminative random fields," Int. J. of Computer Vision, vol. 68(2), pp. 179-201, 2006.

[3] P. Bouthemy, C. Hardouin, G. Piriou, and J. Yao, "Mixed-state auto-models and motion texture modeling," J. of Mathematical Imaging and Vision, vol. 25(3), pp. 387-402, 2006.

[4] F. Tang and V. Prinet, "Computing invariants for structural change detection in urban areas," in Proc. 4th IEEE GRSS/ISPRS Joint Workshop on Remote Sensing and Data Fusion over Urban Areas (URBAN), Paris, France, 2007.

[5] D. Lowe, "Distinctive image features from scale-invariant keypoints," Int. J. of Computer Vision, vol. 60(2), pp. 91-110, 2004.

[6] R. Wiemker, A. Speck, D. Kulbach, H. Spitzer, and J. Beinlein, "Unsupervised robust change detection on multispectral imagery using spectral and spatial features," in Proc. Int. Airborne Remote Sensing Conference, Copenhagen, Denmark, 1997.

[7] T. Crivelli, B. Cernuschi-Frias, P. Bouthemy, and J.-F. Yao, "Mixed-state markov random fields for motion texture modeling and segmentation," in Proc. IEEE Int. Conf. on Image Processing, Atlanta, USA, 2006.

[8] S. Li, Markov random field modeling in image analysis, Springer-Verlag ed., 2001. 